\title{
The Antiproton Ion Collider at FAIR
}

\author{
L. Fabbietti ${ }^{a}$, P. Beller ${ }^{b}$, F. Bosch ${ }^{b}$, M. Cargnellic, \\ T. Faestermann ${ }^{a}$, B. Frankze ${ }^{b}$, H. Fuhrmann ${ }^{c}$, \\ R.S. Hayano ${ }^{d}$, A. Hirtl ${ }^{c}$, J. Homolka ${ }^{a}$, P. Kienle ${ }^{a}$, \\ Ch. Kozhuharov ${ }^{b}$, R. Krücken ${ }^{a}$, H. Lenske ${ }^{e}$, Y. Litvinov $^{f}$, \\ J. Marton ${ }^{c}$, F. Nolden ${ }^{b}$, P. Ring ${ }^{a}$, Y. Shatunov ${ }^{f}$, A.N. Skrinsky ${ }^{f}$, \\ K. Suzuki ${ }^{a}$, V.A. Vostrikov ${ }^{f}$, T. Yamaguchi ${ }^{d}$, \\ E. Widmann ${ }^{c}$, S. Wycech ${ }^{g}$, J. Zmeskal $^{c}$ \\ $a^{a}$ Technische Universität München, Munich, Germany \\ ${ }^{b}$ Gesellschaft fr Schwerionenforschung, Darmstadt, Germany \\ ${ }^{c}$ Stefan Meyer Institut, Vienna, Austria \\ $d$ University of Tokyo, Tokyo, Japan \\ $e$ Justus-Liebig Universität Giessen, Giessen, Germany

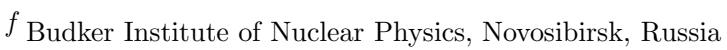 \\ $g$ Andrzej Soltan Institute for Nuclear Studies (IPJ), Warsaw, Poland
}

\begin{abstract}
A novel method [1] is proposed to determine the charge and the matter radii instable and short lived nuclei using an pBar-A collider. The experiment makes use of the appropriately modified electron-ion collider Elise, to collide $30 \mathrm{MeV}$ anti-protons with $740 \mathrm{AMeV}$ ions. The anti-protons are first collected in the $\mathrm{CR}$ ring with $3 \mathrm{GeV}$ energy and then cooled in the RESR ring to 30 $\mathrm{MeV}$. The heavy ions produced in the SFRS are precooled in the CR ring, cooled in the RESR ring to $740 \mathrm{AMeV}$ and fed to the NESR ring. The total pBar-nucleon annihilation cross-section is measured detecting the loss of stored ions and the pBar-n, pBar-p cross-sections detecting the $A-1(Z-1$ or $N-1$ ) nuclei left over after the annihilation, using the Schottcky method. Theoretical predictions show that the annihilation cross-section is proportional to the mean squared radius.
\end{abstract}




\section{Introduction}

The study of the evolution of the density distribution of protons and neutrons has been extensively addressed in the last 20 years as a fundamental tool to investigate the nuclide landscape. Indeed the measurement of matter and charge radii provide the evidence of the occurrence of nuclear halos and neutron skins. While charged radii of short lived nuclei can be deduced in a model independent way using e. g. electron scattering or laser spectroscopy, the neutron radii is so far not straight forward. Experimentally, a variety of efforts have been undertaken to investigate the properties of the neutron-rich exotic nuclei, ranging from high-energy break-up reactions $[2,3]$ and elastic proton scattering $[4,5]$ to inelastic scattering and charge-exchange reactions [6]. Also, anti-protons have been used to probe nuclear size of stable nuclei [7], whereas the atomic capture has been exploited, (see figure 1) but still all the collected data are not consistent among each other and partly model dependent. Most of the existing experimental data refer to stable



Figure 1: Compilation of the difference between proton and neutron radii as a function of the $(N-Z) / A$ ratio. The line refers to a linear fit which parameters are also visible in the picture, the red points to the LEAR experimental data [7].

and long-lived nuclei but the new SFRS facility at FAIR will enable us to extend the investigation to more exotic nuclei.

\section{Expected rates}

Some calculations have been carried out to estimate luminosities and reaction rates. Taken into account an intensity of $10^{9} \mathrm{pBar}$ and about $10^{6}$ ions, whereas this last number accounts for the losses that occur between the SFRS and the NESR ring, the out-coming luminosity is shown in figure 2 as a function of the pBar energy. This luminosity is feasible for nuclei with a half-life $\tau \geq 1 \mathrm{~s}$, due to the time needed to store and cool the ions in the RESR ring. This limit in time translates in a minimal intensity of $10^{5}$ ions. Figure 2 shows also these boundaries on the $N$ versus $Z$ map for $28<Z<52$. On the 

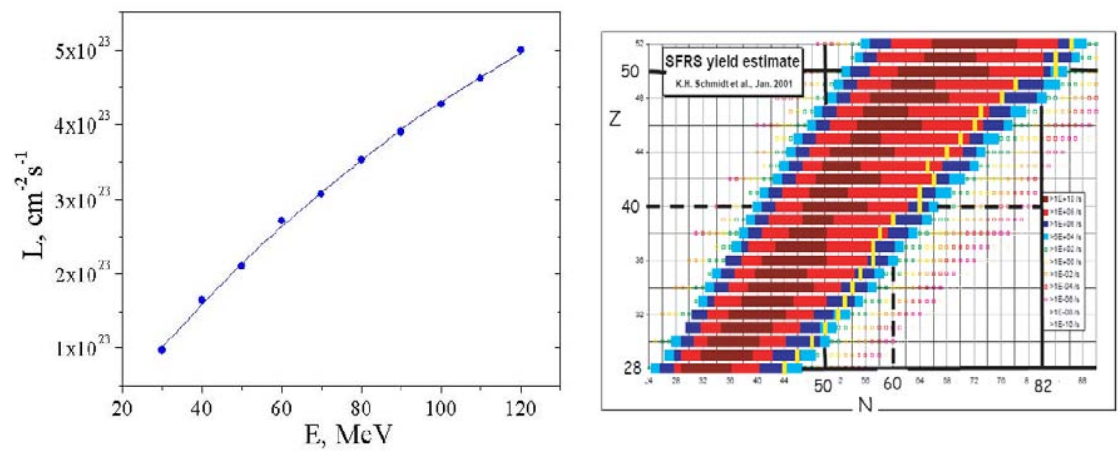

Figure 2: Left: Luminosity of the pBar-A collisions versus pBar energy. Right: SFRS production yields between $\mathrm{Ni}$ and $\mathrm{Sn}$ isotopes. The range of larger symbols indicates production yields above $4.5 \cdot 10^{4}$. The yellow lines indicates the 1 second limit in the half-lives for the neutron-rich nuclei.

neutron-rich side the limits correspond to the yellow lines, while on the neutron-deficient side they correspond to the $N=Z$ line.

Taking for example the ${ }^{55} \mathrm{Ni}$ nucleus, a luminosity of $10^{34} / \mathrm{cm}^{2} / \mathrm{s}$ is reached. Theoretical calculations predict a pBar-nucleon annihilation cross-section of about 1 barn at these energies [11], that translates in about 4.8 annihilation/sec. Further calculations, mentioned later in this text, have determined that this corresponds to $0.72 \mathrm{Z}-1$ and $0.72 \mathrm{~N}-1$ reactions/sec, where these numbers already account for the fraction of A-1 nuclei lost after the annihilation. These numbers lead to a rate of 18000 count/h and if only the statistical error is considered in the radius calculation, a resolution of about $0.01 \mathrm{fm}$ is obtained for the $\Delta r_{n p}$. Table 1 shows the estimated rates for some nuclei.

\section{Annihilation cross-section}

It has been mentioned in the introduction that the radius can be extracted from the measured annihilation cross-section. In order to study this method the pBar absorption has been parametrized using an optical potential, where the real part is taken to be much smaller of the imaginary part. The cross-section has been calculated for the $N i$ chain to study the mass dependence of it. The energy dependence has also been investigated. Because of the strong absorption the annihilation cross-section gains strength only in the nuclear surface, in a short of crescent like distribution with an average radius of about the average of the Wood-Saxon potential. Along the isotopic chain the pBar-p cross-section is slowly varying as a function of the mass number A, but the pBar-n cross-section reflects the rapid change of the neutron density with increasing neutron excess. This can be seen in the bottom panel of figure 3, that shows the annihilation cross-section as a function of $A$. The upper panel of figure 3 shows the $r m s$ proton and neutron radii for the $\mathrm{Ni}$ isotopes with $T_{L A B}=400 \mathrm{MeV}$ calculated using the HFB method. One can see also 


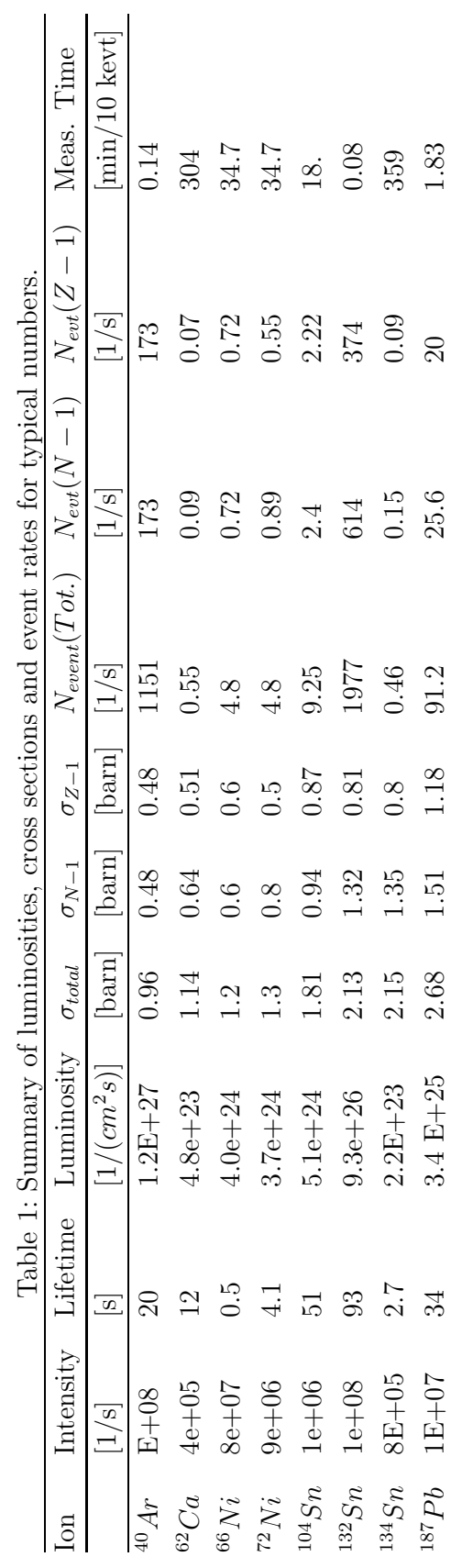



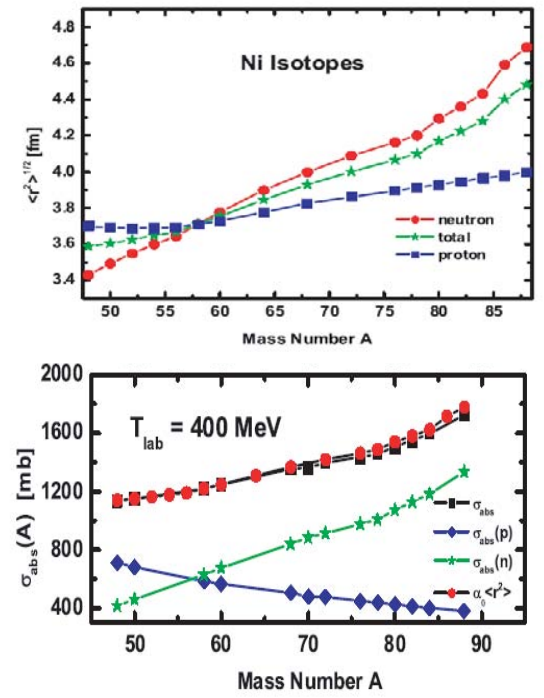

Figure 3: Upper: Root-mean-square (rms) radii for some $\mathrm{Ni}$ isotopes as a function of the mass Number. Bottom: Absorption cross-sections (circles) for pBar annihilation on $\mathrm{Ni}$ isotopes at $T_{L A B}=400 \mathrm{MeV}$ compared to the rms-radii [11].

from this picture the monotonic behavior of the proton radii and the steep increases of the neutrons one. This implies that the measurement of the pBar-A absorption can provide us with data on the existence and evolution of the neutron skin. The relation between the radius distribution and the absorption cross-section is visible in the bottom panel of figure 3 where the total cross-section is overlapped with the radius distribution multiplied with an energy dependent constant $\alpha_{0}$. The rms-radii are in first order directly proportional to the measured absorption cross-section. Hence we can conclude that antiproton annihilation at intermediate energies are an appropriate probe for nuclear sizes and shapes.

A last interesting aspect of this measurement concerns the energy dependency of the cross-section. With increasing energy the magnitude of $\sigma_{a b s}$ decreases but approaches to an asymptotic value close to $T_{l a b}=400 \mathrm{MeV}$. Since the absorption is happening predominantly on the nuclear surface, different energies allow to scan the tail of the wave functions. Figure 4 shows the impact parameter distribution dependency of the absorption cross-section for ${ }^{78} \mathrm{Ni}$ for energies between $50 \mathrm{MeV}$ and $400 \mathrm{MeV}$. One clearly observes the convergence of the cross-sections for higher energies towards the geometric value. This energy scan is made possible by the features of the RESR ring that account for the energy setting of the ions. 


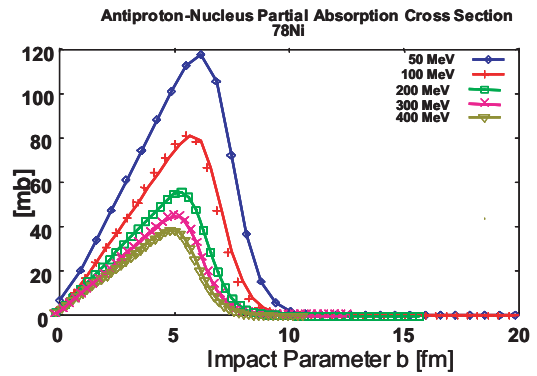

Figure 4: Partial wave absorption cross-sections for anti-protons on ${ }^{78} \mathrm{Ni}$ as a function of the impact parameter of the pBar-A collision for different incident energies from $50 \mathrm{MeV}$ to $400 \mathrm{MeV}[11]$.

\subsection{Missing probability}

The pBar-nucleon annihilation is followed by pions emission, that can eventually cross the A-1 nucleus and interact with it. Only those events in which the pions have missed the nucleus lead to measurable A-1 nuclei in the NESR ring, hence the missing probability $\left(P_{\text {miss }}\right)$ must be taken into account. It can also happen that the pBar annihilates with a deeply bound nucleon, leaving the A-1 nucleus excited. This nucleus can emit a nucleon turning into A-2 nucleus, this probability $\left(P_{d h}\right)$ is also accounted for. Calculations

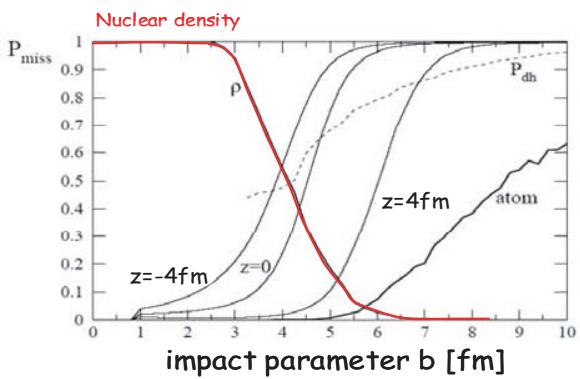

Figure 5: $P_{\text {miss }}$ for ${ }^{58} \mathrm{Ni}$ as a function of the penetration length $z$ of the pBar in the nucleus.

have been carried out [12] to estimate these probabilities for some $\mathrm{Ni}$ and $\mathrm{Sn}$ isotopes, assuming an optical potential and that the nucleus can be seen as a semi-transparent disc where the penetration length $z$ can be differentiate. A relative momentum of $1 \mathrm{GeV} / \mathrm{c}$ between the pBar and the nucleon has been assumed and the results for ${ }^{58} \mathrm{Ni}$ are shown in figure 5. $P_{\text {miss }}$ and $P_{d h}$ are shown together with the nuclear density as a function of the impact parameter $\rho$, the whole reaction plane is described by $(\rho, z)$ whereas $z>0$ denotes the back side of the nucleus. For comparison purposes the total $P_{\text {miss }}$ for the atomic anti-protons is shown. 
The total probability and the average radius seen by the annihilation is calculated integrating over $\rho$ and $z$, the results are collected in table 2. One can see that the relative

Table 2: Comparison between A-1 atoms a la AIC and antiprotonic atoms for ${ }^{58} \mathrm{Ni}$

\begin{tabular}{llll}
\hline$<R>,[\mathrm{fm}]$ & atomic pBar & A-1 tot. & A-1, cold \\
$\Delta R,[\mathrm{fm}]$ & 6.6 & 5.0 & 5.2 \\
$\sigma^{A-1} / \sigma^{\text {total }},[\%]$ & 1.2 & 0.9 & 0.9 \\
\hline
\end{tabular}

rate of $31 \%$ for the AIC reactions is fairly large in comparison with the atomic antiproton case, even if the annihilation is rather peripheral. The rate becomes larges for bigger nuclei, the integrated probability is about $60 \%$ for the ${ }^{132} \mathrm{Sn}$ nuclei.

These factors together with the cross-sections mentioned in section 3 , have been used to estimate the total rates shown in section 2 .

\section{Particle and luminosity measurement}

The A-1 nuclei can be measured in the NESR ring exploiting the Schottcky noise frequency analysis [8]. Each of the nuclei passes trough the pickups distributed along the NESR ring and via the Fast Fourier Transform technique a frequency spectrum is obtained. Since the velocities of all the nuclei are the same after the cooling in the NESR, the revolution frequencies in the ring reflect their mass-to-charge ratios, or for a fixed frequency in ring, the momentum. This method can be applied to ions with $A>60$, since the momentum acceptance of the NESR ring is about $1.75 \%$ in the longitudinal momentum deviation. For the detection of lighter nuclei silicon detectors in a pocket can be placed near the interaction region before the third NESR dipole, that would kick the fragment out of the ring acceptance. The good position and energy resolution of these detectors [9] allow to identify the $Z$ and the $p / q$ ratio of the nuclei.

Another important aspect of the experiment is the measurement of the luminosity, that is later necessary to calculate absolute cross-sections. The time integrated luminosity $L d t$ can be determined detecting the backwards (taking the ion direction as a reference) elastically scattered antiprotons. Calculations have been carried out to estimate the grazing angle for the Rutherford scattering for ${ }^{132} \mathrm{Sn}$ and ${ }^{56} \mathrm{Ni}$. Assuming values of $3.2^{\circ}$ and $2.1^{\circ}$ respectively, a cylindric silicon detector with an angular coverage between $1.5^{\circ}$ and $4^{\circ}$ can enable the calibration.

\section{Physics program and conclusions}

The feasibility of the AIC experiment has been shown as an interesting tool to study the evolution of the matter and charge radii instable and short-lived nuclei.

The experimental program will start with a benchmarking measurements for stable nuclei, 
to establish the method and compare the results with those achieved using the X-Ray spectroscopy. The Sn isotopes constitute a good starting point for the benchmarking and in particular the odd isotopes can be investigated to interpret the pionic isotope shift observed in the deeply bound s-states [10]. Systematic studies of the isotopic and isotonic chains will follow with particular emphases on the odd-even staggering problem. Extended measurements of light nuclei ( $A \approx 20-25)$ near to the drip-line are also foreseen in order to investigate the transition from the halo regime to the neutron skins in heavier nuclei. Last but not least the pBar-nucleon interaction can be investigated quantitatively in the framework of this project.

\section{References}

[1] AIC Technical Proposal GSI (2005).

[2] D. Cortina-Gil et al. Nucl. Phys. A718 (2003) 431.

[3] D. Cortina-Gil et al. Phys. Rev. Lett. 93 (2004)062601.

[4] P. Egelhof, Nucl. Phys. A22 (2003) 254c.

[5] G. D: Alkhazov et al. Nucl. Phys. A712 (2002) 269.

[6] A. Kraznahorkay et al. Phys. Rev. Lett. 82 (1999) 3216.

[7] A. Trcinska, Phys. Rev. Lett. 87 (2001) 082501.

[8] Borer et al., Proc. IX Conf. on High Energy Acceleators, Stanford, 1974.

[9] L. Maier et al. Jahresberich MLL (1999) München, p 97.

[10] K.Suzuki et al. Phys. Rev. Lett 92072302 (2004).

[11] H. Lenske and P. Kienle to be published; this conference.

[12] S. Wycech private communication. 